Case report

\title{
Failure of ultrasound to diagnose a giant ovarian cyst: a case report Themistoklis Mikos ${ }^{1}$, George P Tabakoudis ${ }^{1}$, George Pados ${ }^{1}$, Nikos P Eugenidis ${ }^{2}$ and Efstratios Assimakopoulos ${ }^{1}$
}

\author{
Addresses: ${ }^{1} 1^{\text {st }}$ Department of Obstetrics \& Gynecology, Aristotle University of Thessaloniki, Papageorgiou General Hospital, Periferiaki Odos Neas \\ Efkarpias, 56 429, Thessaloniki, Greece \\ ${ }^{2} 2^{\text {nd }}$ Propedeutic Department of Medicine, Aristotle University of Thessaloniki, Ippokrateion General Hospital, Thessaloniki, Greece \\ Email: TM* - themis.mikos@gmail.com; GPT - ptampako@auth.gr; GP - padosgyn@hol.gr; NPE - mpontis@auth.gr; \\ EA - eassimakopoulos@yahoo.com \\ * Corresponding author
}

Received: 26 March 2009 Accepted: 9 July 2009 Published: 22 July 2009

Cases Journal 2009, 2:6909 doi: 10.4076/1757-1626-2-6909

This article is available from: http://casesjournal.com/casesjournal/article/view/6909

(C) 2009 Mikos et al.; licensee Cases Network Ltd.

This is an Open Access article distributed under the terms of the Creative Commons Attribution License (http://creativecommons.org/licenses/by/3.0), which permits unrestricted use, distribution, and reproduction in any medium, provided the original work is properly cited.

\begin{abstract}
Ultrasonography is the method of choice in the diagnosis of ovarian cysts. In this case report, a cyst of enormous volume (>35 litres) was limiting the application of ultrasound techniques giving the false impression of ascites. A 55-year-old woman was finally diagnosed as having a giant ovarian mucosalserosal cystadenoma of borderline potential after undergoing a total abdominal hysterectomy with salpingo-oophorectomy and excision of the cyst. In the literature, similar conditions have been described with the term 'empty abdomen'.
\end{abstract}

\section{Introduction}

Ovarian cysts rarely grow immense. Ultrasound scan examination permits early detection and appropriate treatment. Occasionally, ovarian cysts reach enormous dimensions without raising any symptom. A few cases of giant ovarian cysts have been sporadically reported in the literature [1-4]. Differential diagnosis with ascites is a major concern during the management of these cysts.

A case of a giant ovarian cyst mimicking ascites in a 59-year-woman is presented. Emphasis is drawn on the initial failure of ultrasound to assist in the diagnosis due to the enormous size of the cyst that hampered the application of ultrasound techniques.

\section{Case presentation}

A 59-year-old, Caucasian (Greek origin), multiparous (Gravida 3, Para2, two spontaneous vaginal deliveries, housewife) was hospitalised in the medical ward complaining of dyspnoea and abdominal distension progressing during the last eight months. She was $1.62 \mathrm{~m}$ high, and she was weighing $73 \mathrm{~kg}$ at admission. She had no significant medical and surgical history, and she did not smoke or drink. Initial abdominal imaging with computed tomography and abdominal ultrasound showed huge ascites and suggested no signs of ovarian or other intraabdominal pathology (Figure 1). Biochemistry was normal except for liver function tests that appeared moderately elevated. Tumor markers were normal except 


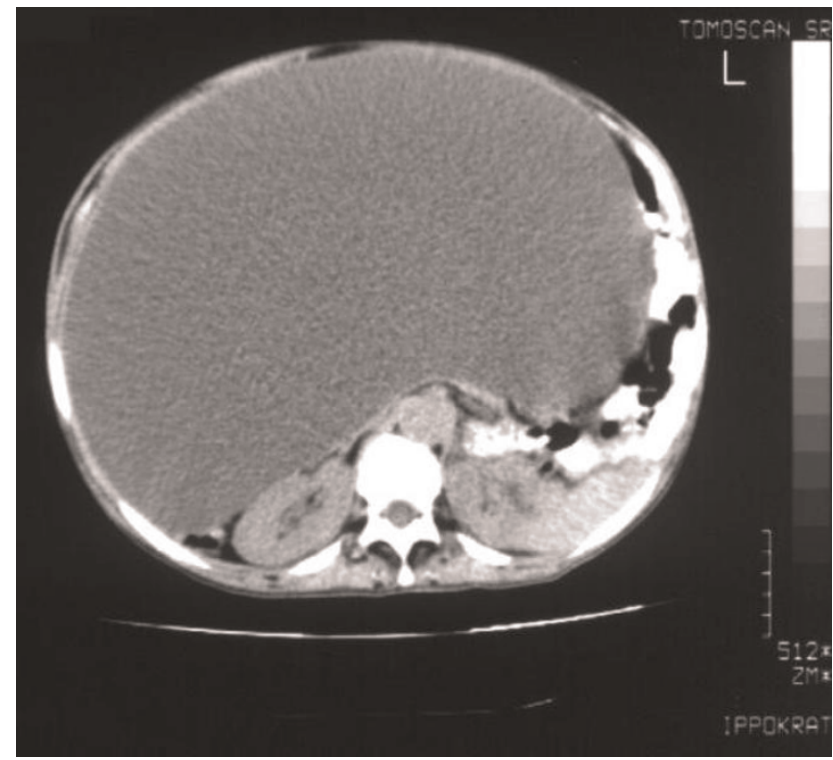

Figure I. Initial computed tomogram of the abdomen of the patient with a giant ovarian cyst mimicking ascites.

CA-125 which was $300 \mathrm{U} / \mathrm{ml}$. Serum-ascites albumin gradient was 1.30 . There was a history of hepatitis $\mathrm{B}$ infection, but at that time the patient was receiving no treatment for the liver. The patient underwent serial drainage of approximately 35 litres of fluid to relieve symptoms. Cytology of the aspirated fluid was normal. The patient was discharged on spironolactone and furosemide and was examined on an outpatient basis. Six months later she was admitted again for recurrence of symptoms. Further drainage removed another 14 litres. Repeat CA-125 was within normal limits. A new sonographic approach (ALOKA, SSD-1700, transabdominal transducer $3.5-5.5 \mathrm{MHz}$ ) after paracentesis revealed normal uterus and left ovary, an increased vascularisation of the right adnexa on the colour flow mode, and the liver characteristically repelled on the back wall of peritoneal cavity, findings compatible with those of a giant ovarian cyst (Figures 2-4). A new computed tomography confirmed the sonographic diagnosis. At subsequent laparotomy the patient underwent excision of the giant cyst and a total abdominal hysterectomy with bilateral salpingo-oophorectomy. Pathology examination showed that the lesion was a mucosal-serosal ovarian cystadenoma of borderline malignant potential. The patient had an uneventful postoperative course and after 3 years of follow up she is doing well.

\section{Discussion}

Giant ovarian cysts mimicking ascites have been rarely reported in the literature. Similar conditions may arise from omentum, mesenterium, or retroperitoneal structures, and the differential diagnosis includes ascites, urinary retention, bladder diverticulum, hydronephrosis, pancreatic pseudocysts, and large uterine tumors [4-10].

It is obvious from the history of the patient that initial sonographic approach failed to diagnose correctly the cyst.
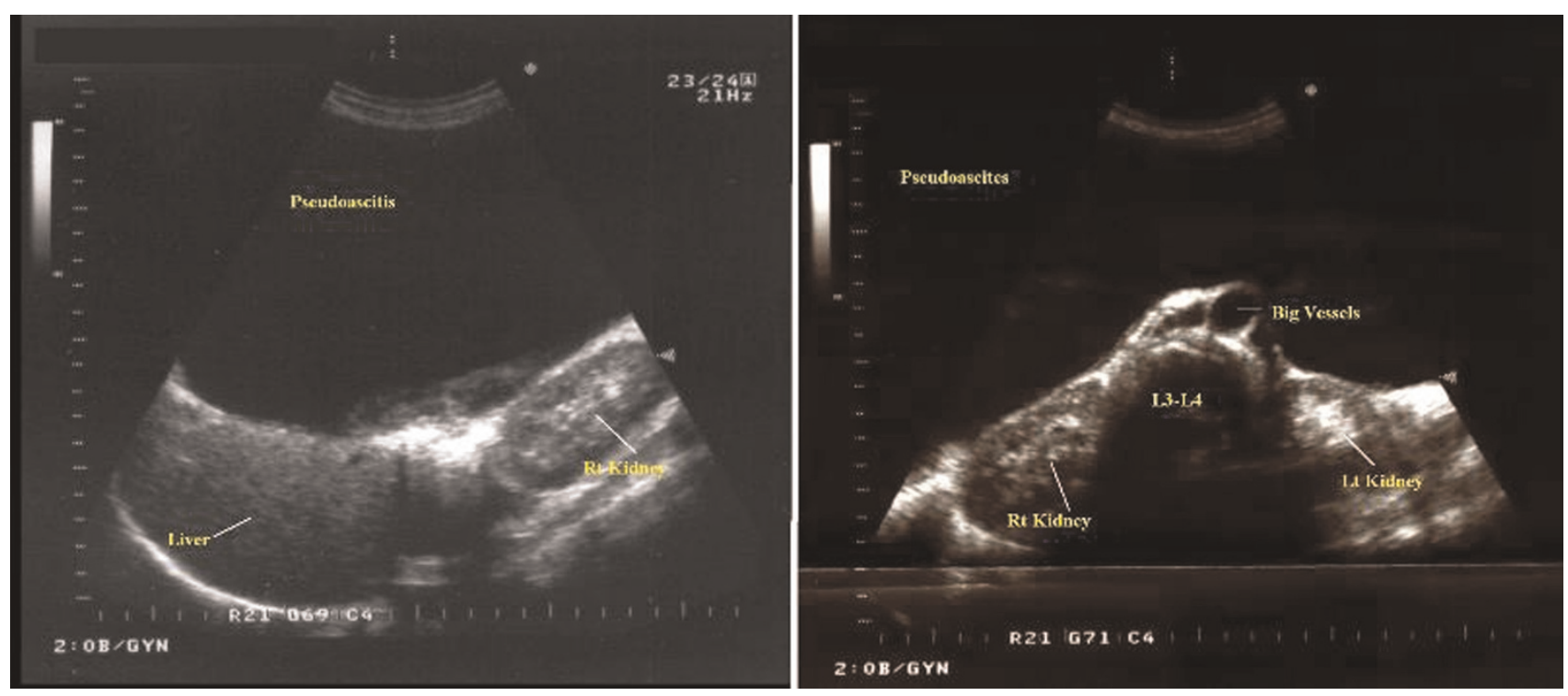

Figure 2. Transabdominal sonogram after paracentesis of the lesion. (Left) The liver and the right kidney are repelled to the posterior wall of the abdomen. The distance between the probe and the abdmominal organs is more than I3 cm. (Right) The large retroperitoneal vessels, the lumbar vertebrae and the two kidneys are seen. 

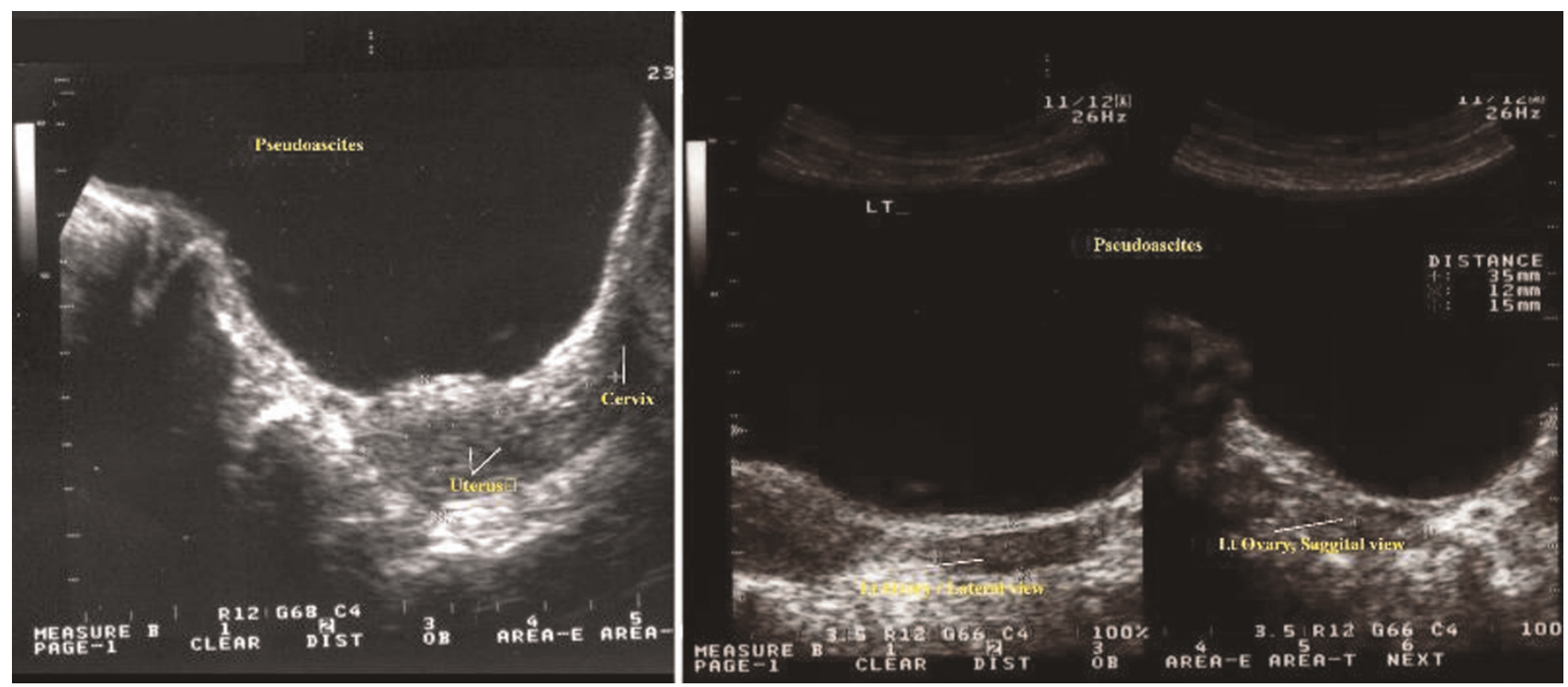

Figure 3. Transabdominal sonogram of (Left) the uterus and (Right) the left ovary of the patient.

However, specific sonographic features that are pathognomonic of giant ovarian cysts were met after paracentesis of the cyst. First, the sonographic appearance of the intraperitoneal organs was highly suggestive of a giant growth. The liver was compressed just above the right kidney (and not free floating), a finding that contradicted to the diagnosis of free intraperitoneal fluid. The absence of other intraperitoneal structures, such as floating bowel

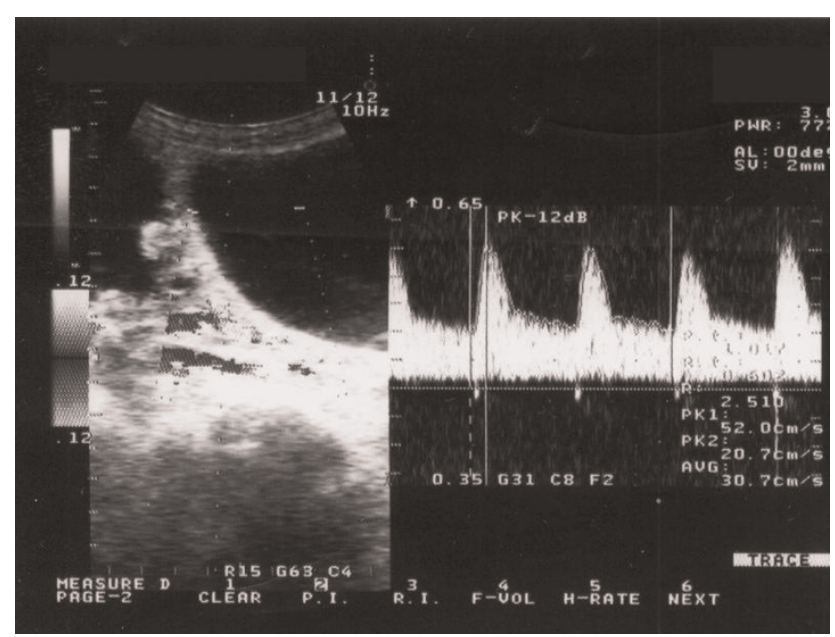

Figure 4. Transabdominal triplex sonogram of the vasculature of the right ovary after paracentesis of the giant cyst. The pulsatility index and the resistance index of the Doppler waveform of the vessels is 1.017 and 0.602 respectively. loops within the abdomen, was another sign of a cystic mass repelling any other abdominal structure away. Second, the use of colour flow was highly suggestive of a neoplastic disorder appearing from the ovary. The colour flow is a sonographic feature that detects movement and is used in the vascular imaging and in producing Doppler waveforms. In the presented case, the colour flow detected a highly vascularised area within the lateral surface of the peritoneal wall. No normal intraoperative organs produce such an intense signal, so the presence of vasculature supplying a huge cyst, probably of ovarian origin, was further suspected. The combination of ultrasound techniques (morphologic assessment, color Doppler flow imaging, and Doppler indexes) have been found to perform well (sensitivity $=84 \%$, specificity $=82 \%$, positive likelihood ratio $=4.69$ ) compared to computed tomography (sensitivity $=81 \%$, specificity $=87 \%$, positive likelihood ratio $=6.81$ ) in the diagnosis of ovarian lesions [11]. Computed tomography had similar findings to the ultrasound suggesting of an ovarian mass repelling the rest of the intraperitoneal organs. Clinical computed tomography images indicate that high accumulation of intraperitoneal fluid can increased the pressure and actually displaces bowel loops to one side of the abdomen and gives a compressed appearance (tense ascites). This is an atypical presentation of ascites that can be easily confused with a mass [12].

The sonographic features described above are pathognomonic of a giant ovarian cyst. The term 'empty abdomen' has been used to describe the sonographic appearance of similar conditions. 


\section{Conclusions}

In conclusion, giant ovarian cysts should always be considered in the differential diagnosis of conditions such as ascites. Two dimensional ultrasound appears to be a cost-effective and accurate technique. The application of extra features such as the colour flow and the Doppler waveforms enhance its diagnostic accuracy. There are cases where drainage of such cysts helps in the application of the sonographic techniques, otherwise the volume of the cysts is a limit to the diagnostic sonographic approach.

\section{Consent}

Written informed consent was obtained from the patient for publication of this case report and accompanying images. A copy of the written consent is available for review by the Editor-in-Chief of this journal.

\section{Competing interests}

The authors declare that they have no competing interests.

\section{Authors' contributions}

TM participated in the diagnosis and the surgical management of the patient, and was a major contributor in writing the manuscript. GT analysed the patient data and participated in the follow up of the patient. GP participated in the surgical management and the inpatient care of the patient, as well as in writing the manuscript. NE performed the gastroenterological examinations and counselling of the patient. EA performed the sonograms and was a major contributor in writing the manuscript. All authors read and approved the final manuscript.

\section{References}

I. Menahem S, Shvartzman P: Giant ovarian cyst mimicking ascites. J Fam Pract 1994, 39:479-48I.

2. Lombardo L, Babando GM: Giant ovarian cyst mimicking ascites. Gastreointest Endosc 1986, 32:245-246.

3. Farinetti A, Buttazzi A, Tazzioli G, Saviano L, Saviano M: Giant ovarian cyst. A case weighing $23 \mathrm{kgr}(50.6 \mathrm{lb})$. Literature review. Minerva Chir 2003, 58:26I-265.

4. Hunter DJ: Management of massive ovarian cyst. Obstet Gynecol 1980, 56:254-255.

5. Semchyshyn S, Strickler RC, Gerulath AH: Giant ovarian cyst in a dwarf. Can J Surg 1977, 20:153-155.

6. Zanini $P$, Cavalca A, Benatti E, Drei B: Benign giant ovarian cystadenoma. Description of a clinical case. Minerva Ginecol 1996, 48:215-219.

7. Rattan KN, Budhiraja S, Pandit SK, Yadav RK: Huge omental cyst mimicking ascites. Indian J Pediatr 1996, 63:707-708.

8. Zamir D, Yuchtman M, Amar M, Shoemo U, Weiner P: Giant mesenteric cyst mimicking ascites. Harefuah 1996, I30:683-684. [In Hebrew].

9. Chen SS: A large retroperitoneal cyst mimicking ascites. A case report. J Reprod Med 1979, 22:26I-263.

10. Grobe JL, Kozarek RA, Sanowski RA, Earnest DL: "Pseudo-ascites" associated with giant ovarian cysts and elevated cystic fluid amylase. Am J Gastroenterol 1983, 78:42I-424.

II. Kinkel K, HricaK H, Lu Y, Tsuda K, Filly RA: US characterization of ovarian masses: a meta-analysis. Radiology 2000, 217:803-8II.

12. Jolles $\mathrm{H}$, Coulam CM: CT of ascites: differential diagnosis. AJR Am J Roentgenol 1980, I35:315-322.

\section{Do you have a case to share?}

\section{Submit your case report today}

- Rapid peer review

- Fast publication

- PubMed indexing

- Inclusion in Cases Database

\section{Any patient, any case, can teach us something}

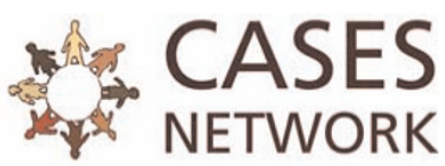

www.casesnetwork.com 